Article

\title{
Evaluation of Recommendation System for Sustainable E-Commerce: Accuracy, Diversity and Customer Satisfaction
}

\author{
Qinglong Li ${ }^{1}$, Ilyoung Choi ${ }^{2}$ and Jaekyeong Kim ${ }^{3, *}$ \\ 1 Department of Social Network Science, KyungHee University, 26, Kyungheedae-ro, Dongdaemun-gu, \\ Seoul, 02453, Republic of Korea; leecy@khu.ac.kr \\ 2 Graduate School of Business Administration, KyungHee University, 26, Kyungheedae-ro, Dongdaemun- \\ gu, Seoul, 02453, Republic of Korea; choice102@khu.ac.kr \\ 3 School of Management, KyungHee University, 26, Kyungheedae-ro, Dongdaemun-gu, Seoul, 02453, \\ Republic of Korea; jaek@khu.ac.kr \\ * Correspondence: jaek@khu.ac.kr; Tel.: +82-2-961-9355 (F.L.)
}

\begin{abstract}
With the development of information technology and the popularization of mobile devices, collecting various types of customer data such as purchase history or behavior patterns became possible. As the customer data being accumulated, there is a growing demand for personalized recommendation services that provide customized services to customers. Currently, global e-commerce companies offer personalized recommendation services to gain a sustainable competitive advantage. However, previous research on recommendation systems has consistently raised the issue that the accuracy of recommendation algorithms does not necessarily lead to the satisfaction of recommended service users. It also claims that customers are highly satisfied when the recommendation system recommends diverse items to them. In this study, we want to identify the factors that determine customer satisfaction when using the recommendation system which provides personalized services. To this end, we developed a recommendation system based on Deep Neural Networks (DNN) and measured the accuracy of recommendation service, the diversity of recommended items and customer satisfaction with the recommendation service. The experimental results of is the study showed that both recommendation system accuracy and diversity would have a positive effect on customer satisfaction. These results can further improve customer satisfaction with the recommendation system and promote the sustainable development of e-commerce.
\end{abstract}

Keywords: expectancy disconfirmation theory; customer satisfaction; e-commerce personalized service; recommendation system; deep neural network

\section{Introduction}

With the development of information technology and the popularization of mobile devices, the e-commerce market continues to grow. Although many new products are released to satisfy consumer's needs, customers are spending too much time selecting their preferred products. Therefore, the importance of personalized recommendation services has emerged. Global companies such as Amazon [1], Netflix [2] and Google [3] are offering various services using the recommendation system to pursue the sustainable development of e-commerce [4]. Recommended the items or services that suit customers' interests not only can improve customer satisfaction by reducing customers' exploring efforts but also increase item sales $[5,6]$. Notably, the recommendation system that recommended items or services using customer's purchase history data helps them make decisions among their various alternatives [7].

The previous study's recommendation systems focused on enhancing the performance of the recommendation system using customers' purchasing history or preference $[8,9]$. The performance of the recommendation system was mainly measured by recommendation accuracy and the diversity 
of recommended items. Recommendation accuracy shows how well the customer's actual preference and predicted preference fit and recommended diversity shows if the customers are willing to recommend the products that recommended by the system and have not previously been purchased to others [8]. A general recommendation system study aims to increase the predictive accuracy of the recommendation system [10-15]. However, a study suggests that even if the recommendation system is accurate, when customers were recommended with the same item every time, their satisfaction or reliability is likely to decrease [16]. Other studies suggest that pursuing diversity while maintaining a certain level of recommendation accuracy in the recommendation system increase customer satisfaction [17]. Thus, although the study of recommendation systems focuses on enhancing the performance of the model, customer satisfaction with the recommendation system is just as important as the improvement of the recommended system performance. Nonetheless, not many studies have considered the performance of the recommendation systems and their relation with customer satisfaction.

Among the existing studies of the recommendation systems, one of the most representative analysis technique used is Collaborative Filtering [18]. Collaborative Filtering is a technique that recommends items that are suitable for customers based on their similar neighbors' preferences and purchasing history $[19,20]$. However, Collaboration Filtering has the issue of Cold Start, which is unpredictable for new customers due to a lack of historical data on their past purchases, and the issue of the First Start, which cannot be recommended until someone reflects their preferences [21]. In addition, the scalability of the model could also become an issue as customers' purchasing data are accumulating continually. Collaborative Filtering techniques may cause problems with poor accuracy of models if they use all customer data [21]. Many studies were conducted to supplement such issues as data sparsity and scalability $[13,21]$. Recently, deep learning techniques show high performance in image processing or natural language processing areas, has been received attention [22-24]. Recently, many researched has been proposed to apply deep learning techniques to recommendation systems [25-29]. For example, recommendation was proposed using the Recurrent Neural Network technique in an environment where there is no customer preference information available for recommendation [30]. In other studies shown that in environments with customer preference data, it is possible to use Embedding methods and Deep Neural Networks to improve recommended accuracy over Collaborative Filtering methods [31].

Therefore, in this study, in order to overcome data sparsity and scalability issues in recommendation systems, we developed a Deep Neural Network-based recommendation system and identified factors that could affect the level of customer satisfaction. To explore this question, we first employ the expectancy disconfirmation theory (EDT) [32]. According to expectancy disconfirmation theory, the customer's satisfaction depends on the expected level before the purchase of the item and the quality difference after the purchase [33-36]. In other words, if the quality of the goods after purchase is higher than the expected level before purchase, the customer will be satisfied. Conversely, if the quality of the goods after purchase is lower than the expected level before purchase, the customer will not be satisfied. Second, for the experiment of this study, we develop a Deep Neural Network-based recommendation system and measured the accuracy, diversity and customer satisfaction through a series of experiments with real dataset. Finally, we statistically analyzed the experiment output data to identify which factors could affect the level of customer satisfaction.

Our experiment results indicate that accuracy and diversity positively could have an effect on customer satisfaction. Thus, we claim that the accuracy of recommendation systems and the diversity of recommended items are important for improving customer satisfaction.

\section{Literature Review}

\subsection{Recommendation System}

A recommendation system provides the customer with proper items or services based on the customer's purchasing history and their deduced preference [37]. Previous research on most 
recommendation systems mainly are based on Collaborative Filtering and Content-based Filtering as shown in Figure 1 [38].

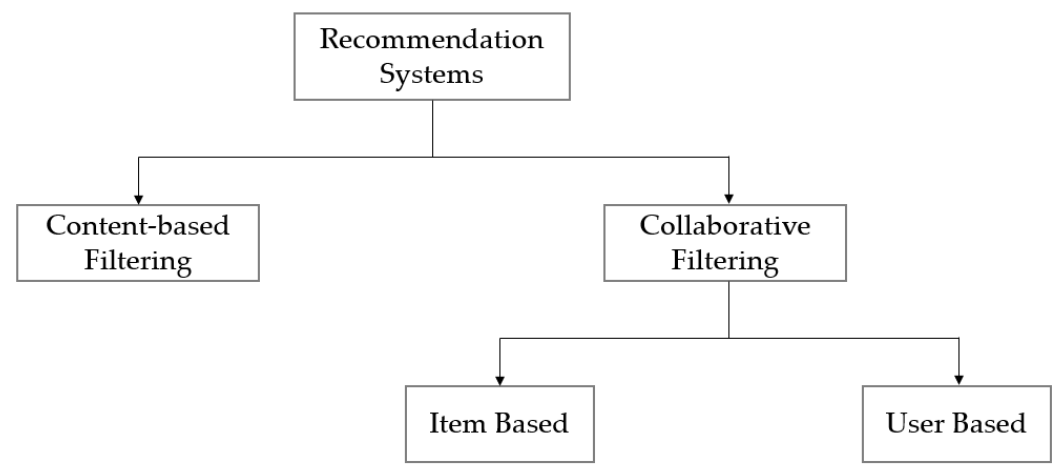

Figure 1. The types of Recommendation Systems

The Collaborative Filtering-based recommendation system is to predict preferences by calculating similarities between customers or items [18]. This recommended method basically recommends items based on customers' past purchasing history and preferences. However, his method has a Cold-Star issue where there is not enough data available to measure similarity and the customer's preferences [21]. Furthermore, there is also a First-Star issue where customers' preferred items are not recommended because they have not yet been purchased [21].

The Content-based Filtering recommendation system is a method to analyze the contents of a item and analyze the similarity between items and customer preferences, and to recommend suitable items to the customer [39]. This method does not cause the First-Rate issue such as the Collaborative Filtering method, because customers are recommended for items of a similar category to the attributes of the preferred items [21, 40]. However, it fails to reflect the taste or preferences of other customers as it recommends items with high similarity based on the customers' purchasing history. So, this method has an Over Specialization issue that items are similar to those purchased previously is recommended [41].

\subsection{Deep Neural Network}

Deep Neural Network refers to a network of two or more hidden layers between the input and output layers as shown in Figure $2[42,43]$. This method uses sophisticated mathematical modeling to solve complex problems. Traditional machine learning algorithms are made up of one input and output layer, but the Deep Neural Network contains multiple hidden layers, so it can learn various nonlinear relationships [44]. Thus, Deep Neural Network has the advantage of being able to identify the potential structure of data. However, the existing deep neural network has occurred Vanishing Gradient issues if layers increase, and excessive learning of models leads to problems such as overfitting or low learning speed.

Input Layer Hidden Layer $1 \quad$.... $\quad$ Hidden Layer N Output Layer

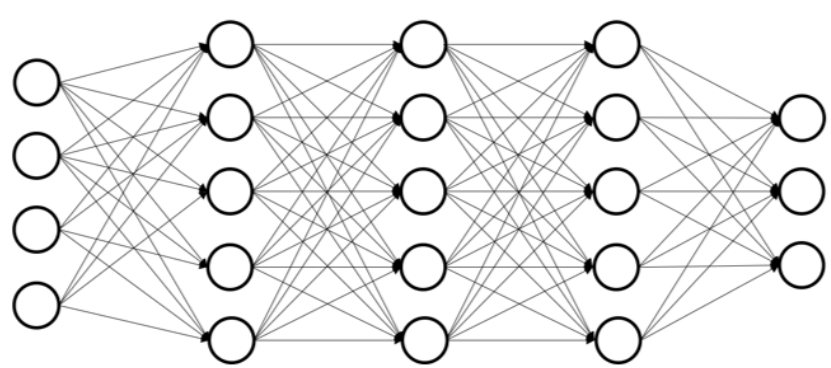

Figure 2. Deep Neural Network Basic Structure 
To solve the Vanishing Gradient in Deep Neural Network, the University of Toronto, professor Jeffrey Everest Hinton proposed a ReLU (Rectified Linear Units) activated function [45]. This function is currently the most commonly used activation function, emerging as an alternative to the existing Sigmoid activation function. Then, to solve the Over Fitting issue in the learning process Methods were proposed such as these Mini Batch, Dropout [46]. Furthermore, GPU specialized in vector operations can process large sizes of data quickly, greatly improving the overall learning speed of the model [43]. Currently, the issue that Deep Neural Networks had is solved and are used in different areas such as image processing and natural language processing [47-49].

\subsection{Expectancy Disconfirmation Theory}

Expectancy disconfirmation theory (EDT) is known as the theory that describes the process of determining customer satisfaction with items and services [32]. According to the theory, if the performance for items and services is higher than expected the customer becomes satisfaction, and if the performance is lower than expected, the customer becomes dissatisfaction [32]. In other words, higher performance than a customer's expectations for a special item or service is positive disconfirmed, but a lower performance than the customer's expectations is negative disconfirmed. If the positive disconfirmed is increasing that may increase customer satisfaction and the negative disconfirmed is increasing may increase in customer dissatisfaction [50]. Especially, negative disconfirmed are important factors to consider, not just dissatisfaction, but also the customers may have negative results such as moving to other items or services or discontinuing purchases [51, 52].

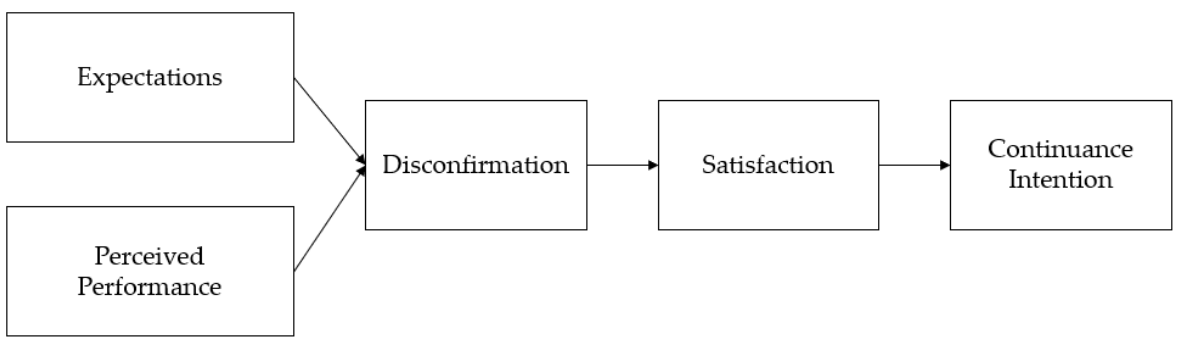

Figure 3. Expectancy Disconfirmation Theory model

Expectancy disconfirmation theory is used in studies to identify the impact on the intention of continuous use of information systems (IS) in the latest technology or online environment [53, 54]. As shown Figure 3 IS continuance intention is influenced by customer satisfaction, which is determined by the difference between perceived quality and expectation levels. In conclusion, customer satisfaction has a positive effect on repurchase intentions and word-of-mouth.

This Expectancy disconfirmation theory can also be applied to the recommendation system. At this time, the expectation of item or service quality that the customer had is compared to the actual item or service quality perceived by the customer through the recommendation system. In the online store, many customers post star rating to the items that they have purchased. Star ratings are important for predicting initial expectation levels for recommended items because the recommendation system predicts customer's purchase scores based on star ratings. Additionally, star ratings are important in measuring performance after purchase because high and low ratings indicate positive and negative views of items [55]. Therefore, we compute disconfirmation as the average of the differences between predicted ratings and actual ratings. The disconfirmation is computed as follows:

$$
\text { Disconfirmation }=\frac{1}{m} \sum_{i=1}^{n}\left(y_{i}-f_{i}\right)
$$

where $m$ is the total number of the recommended items, and $y_{i}$ and $f_{i}$, are the actual star ratings and the predicted star ratings, respectively. 


\subsection{Accuracy and Diversity Metrics of recommendation system}

The performance of recommendation systems can be measured with accuracy and diversity. To evaluate the rating prediction model accuracy, the difference between prediction rating and the actual rating is compared. In this study, the model accuracy was measured based on the mean absolute error (MAE). The mean absolute error (MAE) is computed as follows [8, 56]:

$$
M A E=\frac{\sum\left|p_{i, j}-r_{j, j}\right|}{N}
$$

where $N$ is the total number of recommended items, $p_{i, j}$ and $r_{j, j}$ are the predicted star rating and the actual star rating by customer $i$ for item $j$. In other words, an absolute value is taken for the difference between the predicted star rating and the actual star rating and then the sum of them is divided by the total number of recommended items. The mean absolute error (MAE) is the mean of the absolute value of the error, and regardless of the magnitude of the error, it will be given the same weight.

Recently, most studies have suggested measuring diversity as it has been identified that there is a limit to focusing only on improving the accuracy of the recommendation system. Lathia et al [17] proposed to measure the diversity of recommended items as follows:

$$
\operatorname{Diversity}\left(L_{1}, L_{2}, \mathrm{~N}\right)=\frac{\left|L_{2}-L_{1}\right|}{N}
$$

Where recommended list $L_{1}$ is recommended at one point and $L_{2}$ is recommended at the next point to customers. Next, in the $L_{2}$ recommended list, divide the number of items not on the $L_{1}$ recommended list by $N$.

\section{Hypotheses Development}

Customer satisfaction includes the customer's assessment after purchasing the item or service [57-59]. It is important for companies to satisfy customers because it is likely that customers who are satisfied with their items and services will repurchase and recommend those to the people around them.

The previous studies of the recommendation systems have been done mainly in a way to improve the accuracy [10-14]. In fact, most studies have suggested algorithms that improve the recommendation system accuracy. Research shows that customer satisfaction with a recommendation system can change depending on how accurately recommend items and services for customers [60-62]. Moreover, if recommendations are made to suit the customer's preference, the customer can form a positive attitude toward the recommendation system. In other words, accurate recommendations increase the likelihood that customers will find items that suit their preferences, which in theory increases customer satisfaction. Therefore, the hypothesis is as follows:

Hypothesis1 (H1): Accurate recommendations as a function of the size of customer dataset positively influence customer satisfaction

It is known that if the recommendation system is highly accurate the customer satisfaction level is high [37]. However, even if the recommendation system is highly accurate, the satisfaction or reliability of the recommendation system will decrease if the customer received the recommendation service composed of the same items every time. More studies have claimed that it is very important to provide a variety of items or services to customers while maintaining a certain level of accuracy [17]. Other studies argue that a more diverse list of recommendations increases the probability that a customer will purchase the items. [63-66]. As a result, more various recommended items improve customer satisfaction. Therefore, the hypothesis is as follows: 
Hypothesis2 (H2): Diverse recommendations as a function of the size of customer dataset positively influence customer satisfaction

\section{Experiments}

To test the hypothesis, we developed a Deep Neural Network-based recommendation system, which is free of sparsity and scalability issues of traditional recommendation systems. And we measured the accuracy, diversity and customer satisfaction of the system through a series of experiments with a real dataset. Then, we statistically analyze the experiment result data to identify which factors could affect customer satisfaction. The overall flow chart is shown in Figure 4.

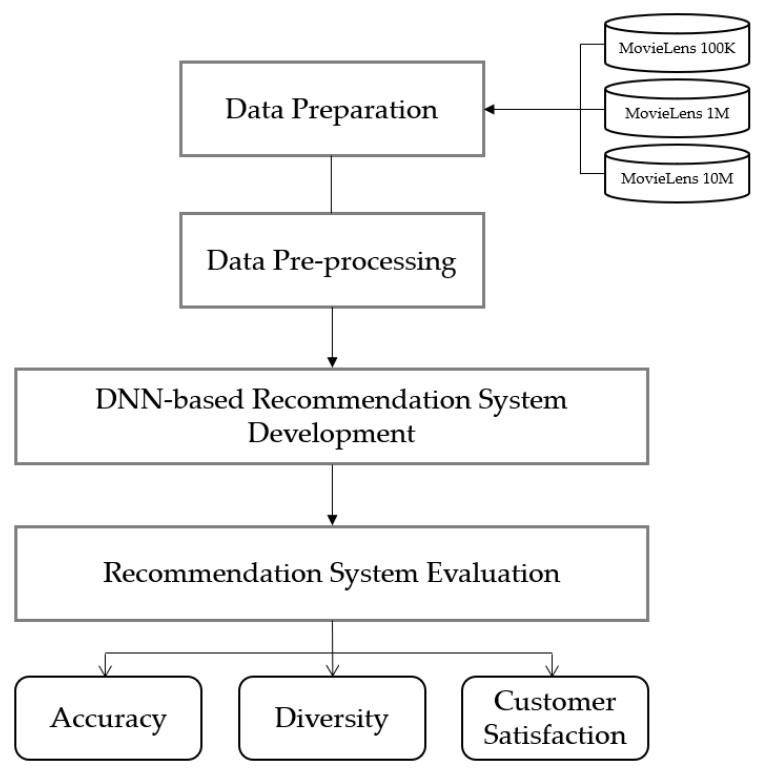

Figure 4. Flow chart of method steps

\subsection{Datasets Description}

For experiments, we use three datasets: MovieLens 100K, MovieLens 1M, MovieLens 10M from GroupLens. All datasets contain star ratings on items (1 to 5 stars). The first dataset, MovieLens 100k dataset contains 100,000 ratings from 943 users on 1,682 movies and the density is $6.30 \%$. The second dataset, MovieLens $1 \mathrm{M}$, with 1 million ratings from 6,040 users on 3,706 items and the density is $4.47 \%$. The third dataset, MovieLens $10 \mathrm{M}$ dataset contains $10,000,054$ ratings by 69,878 users on 10,677 items and the density is $1.34 \%$. In this study, experiments were conducted by selecting items with more than 50 ratings and users with more than 25 ratings. A summary of dataset statistics is shown in Table 1.

Table 1. Descriptive statistics of the MovieLens datasets.

\begin{tabular}{ccccc}
\hline Dataset & \#users & \#items & \#ratings & \#density \\
\hline MovieLens 100K & 943 & 1,682 & 100,000 & $6.30 \%$ \\
MovieLens 1M & 6,040 & 3,706 & $1,000,209$ & $4.47 \%$ \\
MovieLens 10M & 69,878 & 10,677 & $10,000,054$ & $1.34 \%$ \\
\hline
\end{tabular}

\subsection{Implementation Details}

To test our research hypothesis and in order to overcome data sparsity and scalability issues, we developed a Deep Neural Network (DNN)-based recommendation system as shown in figure5. Then we measured the accuracy and diversity by MAE and diversity metrics proposed by Lathia et al [17] , respectively, which are shown in equation (2) and (3). A previous study used questionnaires to 
measure customer satisfaction with the recommendation system $[67,68]$. In this study, we measured disconfirmation-based customer satisfaction through a series of experiments. Here, disconfirmation is computed as the average of the differences between predicted ratings and actual ratings. Experiments used $80 \%$ as a training dataset and $20 \%$ as a test dataset. A training dataset used to train the recommendation system, and the test dataset is used to predict the ratings and recommends Top5 lists with the highest ratings for the customer.

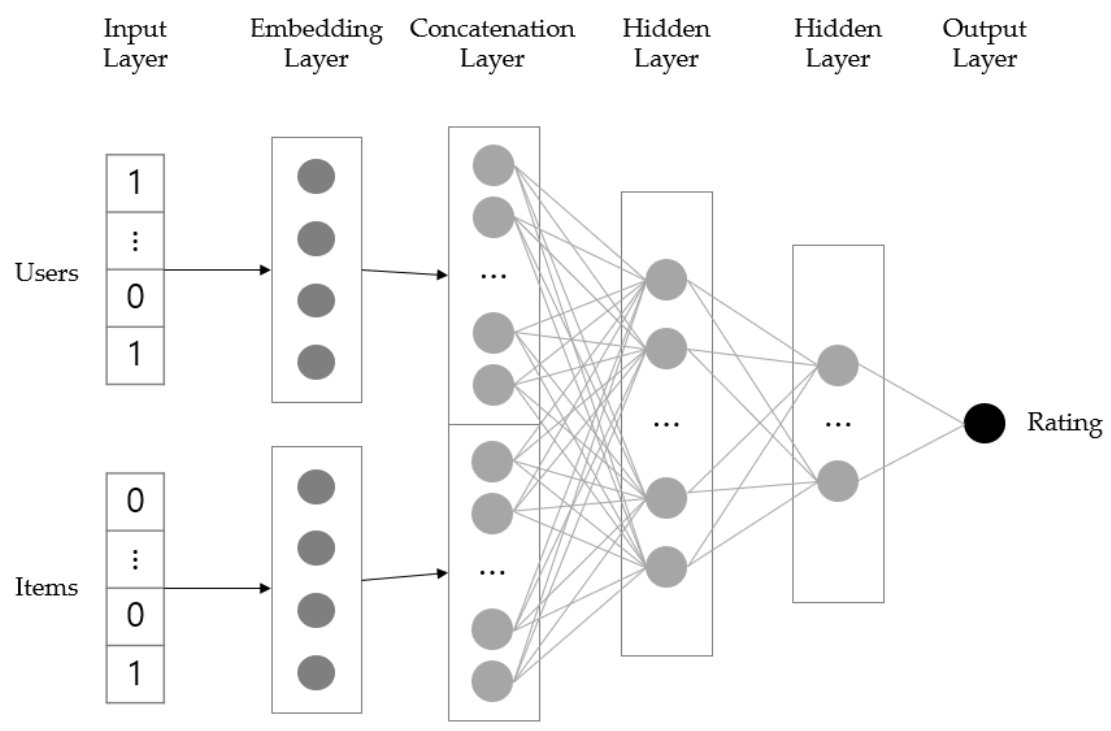

Figure 5. Deep Neural Network based-Recommendation System

To training the Deep Neural Network-based recommendation system, the dataset consisting of user ID, movie ID, and ratings are converted to user embedding vector, movie embedding vector, and ratings to form a training dataset. The Deep Neural Network-based recommendation system used in this study is shown in Figure 5. We develop the system based on Keras 2.2.4 and test it on an NVIDIA GEFORCE RTX 2080 Ti GPU. This recommendation system is trained by estimating the relationship between the latent vector of the user and the latent vector of the item. Therefore, we separately construct a 20-dimensional embedding vector model for the user ID and a 10-dimensional embedding vector model for the movie ID. Then, the Deep Neural Network model was constructed that combines the two models in the input phase of the fully connected layer.

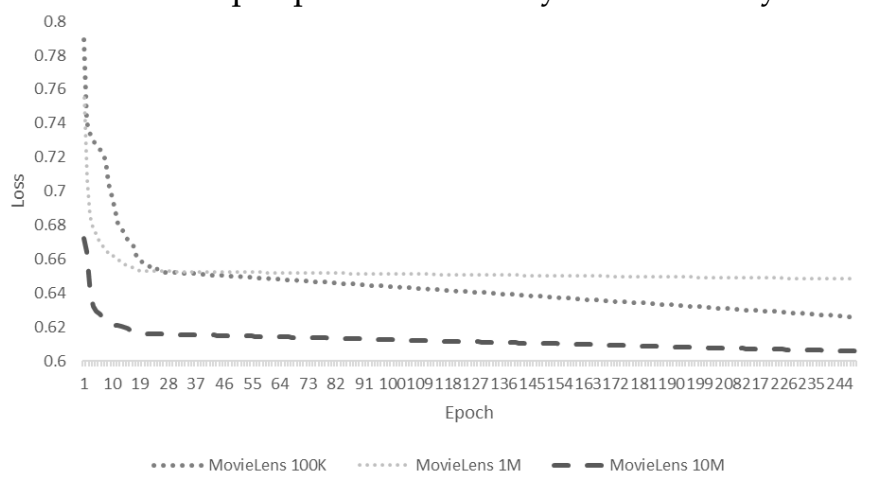

Figure 6. Comparison Training Loss Graph

The input layer receives the training dataset and outputs it without any conversion process. The user models and item models output data in 10-dimensional vectors and 20-dimensional vectors through the embedding layer. The user model and item model output vector are connected, and it combined into a 30-dimensional vector and entered a fully connected layer. The hidden layer of the fully connected layer consists of three nodes and converts the data from a 30-dimensional vector into a 10-dimensional vector to output. As a hidden layer activation function, it is used the RELU 
(Rectified Linear Unit). The output layer consists of one node and outputs 10-dimensional vector data as a 1-dimensional value. The experiment was repeated up to 250 times until the loss value remained unchanged as shown in Figure 6. Then the optimization function is used Adam and as the loss function, it is used MAE. After training the Deep Neural Network model, the user embedding vector and item embedding vector of the test dataset are inputted to predict the rating of the item and recommends Top-5 lists with the highest ratings for the customer.

\subsection{Results}

The mean and standard deviation for accuracy, diversity, and customer satisfaction at MovieLens 100K, MovieLens 1M, MovieLens 10M are listed in Table 2. The mean value for accuracy and diversity was between 0.655 and 0.722 and between 0.477 and 0.570 , respectively. Furthermore, the mean value of customer satisfaction was between 0.144 and 0.204 . The highest value of accuracy is at a MovieLens $10 \mathrm{M}(0.655)$ and the lowest value of accuracy at a MovieLens $100 \mathrm{~K}(0.722)$. The highest value of diversity is at a MovieLens $1 \mathrm{M}(0.570)$ and the lowest value of diversity is at a MovieLens $100 \mathrm{~K}(0.477)$. The highest value of customer satisfaction is at a MovieLens $1 \mathrm{M}(0.204)$ and the lowest value of customer satisfaction is at a MovieLens $100 \mathrm{~K}(0.165)$. Especially, items with highly predicted ratings are recommended regardless of the actual purchase. So, customer satisfaction is positive because it is defined as the average of the differences between predicted ratings and actual ratings.

Table 2. Descriptive statistics of accuracy, diversity, and customer satisfaction.

\begin{tabular}{cccc}
\hline Datasets & Variables & Mean & Standard Deviation \\
\hline \multirow{3}{*}{ MovieLens 100K } & Accuracy & 0.722 & 0.415 \\
& Diversity & 0.477 & 0.296 \\
& Customer Satisfaction & 0.165 & 0.620 \\
\hline \multirow{3}{*}{ MovieLens 1M } & Accuracy & 0.673 & 0.399 \\
& Diversity & 0.570 & 0.292 \\
& Customer Satisfaction & 0.204 & 0.555 \\
\hline \multirow{3}{*}{ MovieLens 10M } & Accuracy & 0.655 & 0.368 \\
& Diversity & 0.522 & 0.295 \\
& Customer Satisfaction & 0.144 & 0.525 \\
\hline
\end{tabular}

To test the research hypothesis, we performed multiple regression analyses under the three Datasets. Table 3 summarizes the results of multiple linear regressions for hypotheses $\mathrm{H} 1$ and $\mathrm{H} 2$. The table shows the unstandardized regression coefficient, the standardized regression coefficient, $\mathrm{t}$ value, tolerance, and variance inflation factor (VIF) of each predictor and R2, adjusted R2, F, and Durbin-Watson of each model in linear regression analysis. Under the three Datasets, each model is statistically significant ((MovieLens 100K) F=80.798, $\mathrm{p}<0.01$, (MovieLens 1M) F=1140.951, $\mathrm{p}<0.01$ and (MovieLens 10M) F=13030.16, $\mathrm{p}<0.01$ for three Dataset predicting $16.8 \%, 29.1 \%$ and $29.7 \%$ of the variance in customer satisfaction). Moreover, there is no multicollinearity between accuracy and diversity under the three Datasets ((MovieLens 100K) tolerance $=.999$ and VIF $=1.001$, (MovieLens 1M) tolerance $=.999$ and VIF $=1.001$, and (MovieLens $10 \mathrm{M}$ ) tolerance $=.999$ and VIF $=1.001$ ). The results show that accuracy and diversity positively and significantly affect the disconfirmation $(\mathrm{p}<0.01)$, therefore supporting hypothesis 1 and hypothesis 2 . These results suggest that both recommended accuracy and diversity are important for customer satisfaction.

Table 3. Results of multiple regression analysis.

\begin{tabular}{ccccccc}
\hline Datasets & $\begin{array}{c}\text { Dependent } \\
\text { Variable }\end{array}$ & $\begin{array}{c}\text { Unstandardized } \\
\text { Beta }\end{array}$ & $\begin{array}{c}\text { Standardized } \\
\text { Beta }\end{array}$ & t-value & Tolerance & VIF \\
\hline MovieLens & Accuracy & .391 & .262 & $9.979^{* *}$ & .999 & 1.001 \\
$100 \mathrm{~K}$ & Diversity & .674 & .321 & $8.121^{* *}$ & .999 & 1.001 \\
\hline
\end{tabular}


Error! Unknown switch argument. of 13 $R^{2}=0.168$, Adjusted $R^{2}=0.165, \mathrm{~F}=80.798^{* *}$, Durbin-Watson=2.001

\begin{tabular}{|c|c|c|c|c|c|c|}
\hline \multirow{3}{*}{$\begin{array}{c}\text { MovieLens } \\
1 \mathrm{M}\end{array}$} & Accuracy & .597 & .430 & $38.036^{* *}$ & .999 & 1.001 \\
\hline & Diversity & .603 & .317 & $28.017^{* *}$ & .999 & 1.001 \\
\hline & \multicolumn{6}{|c|}{$R^{2}=0.292$, Adjusted $R^{2}=0.291, \mathrm{~F}=1140.951^{* *}$, Durbin-Watson=2.016 } \\
\hline & Accuracy & .581 & .408 & $120.673^{* *}$ & .999 & 1.001 \\
\hline MovieLens & Diversity & .622 & .350 & $103.430^{* *}$ & .999 & 1.001 \\
\hline
\end{tabular}
$10 \mathrm{M}$ $R^{2}=0.297$, Adjusted $R^{2}=0.297, \mathrm{~F}=13010.396^{* *}$, Durbin-Watson=1.992

$$
{ }^{* *} \mathrm{p}<0.01,{ }^{*} \mathrm{p}<0.05
$$

Also, a one-way analysis of variance (ANOVA) was conducted to determine whether there was a significant difference in customer satisfaction under the three datasets. The Scheffe Post Hoc Test was used to identify multiple comparisons of group means. The results presented in Table 4 indicated that there was a significant customer satisfaction difference between the dataset. $(\mathrm{F}=34.088$, Sig. $=$ 0.000). The significant mean difference was found between MovieLens $1 \mathrm{M}$ and MovieLens 10M (mean difference $=-0.609$, Sig. $=0.000$ ), which indicates that customer satisfaction is related to diversity when the accuracy is somewhat constant.

Table 4. One-way ANOVA of customer satisfaction.

\begin{tabular}{cccccc}
\hline $\begin{array}{c}\text { One-Way } \\
\text { ANOVA }\end{array}$ & $\begin{array}{c}\text { Sum of } \\
\text { Squares }\end{array}$ & Df & Mean Square & F & Sig. \\
\hline Between Group & 19.077 & 2 & 9.539 & 34.088 & .000 \\
\hline Within & 19022.218 & 67978 & 0.280 & & \\
Group & 19041.296 & 67980 & & & Sig. \\
\hline Total & Feature & Mean & Std.Error & 0.148 \\
\hline \multirow{2}{*}{ MovieLens 100K } & MovieLens 1M & Difference & 0.199 & 0.507 \\
\hline \multirow{2}{*}{ MovieLens 1M } & MovieLens 10M & 0.218 & 0.188 & 0.148 \\
\hline \multirow{2}{*}{ MovieLens 10M } & MovieLens 100K ** & 0.039 & 0.199 & 0.007 \\
\hline
\end{tabular}

\section{Discussion and Conclusions}

A recommendation system is a critical tool for e-commerce companies to pursue sustainable growth. Therefore the global companies such as Amazon [1], Netflix [2] and Google [3]are offering recommendation services to their customers to gain a competitive advantage. However, there are not only trade-offs between recommendation accuracy and diversity of recommended items but also continuing debates over which factor, accuracy or diversity, have a more significant impact on customer satisfaction. Thus, we investigated which factors affect customer satisfaction through statistical analyses of a series of experiments performed with a Deep Neural Network-based recommendation system.

This study provides the following implications for the performance aspects of the recommendation systems and customer satisfaction. First, we used the expectancy disconfirmation theory (EDT) to measure customer satisfaction with Deep Neural Network-based recommendation systems for the first time. In particular, the recommended accuracy and recommended diversity of the recommendation system were measured using a real dataset, so disconfirmation was calculated as an indicator for measuring customer satisfaction. Second, we identified the impact of dataset size 
on recommended accuracy and diversity. The larger the dataset size, the recommended accuracy increase, but it is not sure about the diversity metric because it increases and decreased according to the increase of dataset size. Third, we identified factors that affect customer satisfaction. While recommendation accuracy has continued to increase, and diversity has decreased, then results that all have a positive impact on customer satisfaction. This is consistent with the results of previous studies in which the customer was satisfied by various items recommended while maintaining a certain level of accuracy [17].

As a result, these results provide e-commerce companies with the following insights. First, it is possible to increase customer satisfaction by recommending various items while maintaining a certain level of constant accuracy. Through, suppliers can increase sales by offering a variety of items that meet customer preferences. Second, the more customer datasets have high recommendation accuracy, but the diversity and satisfaction levels are rather reduced. Therefore, when customer data is accumulated to some extent, it should be managed regularly.

There are several limitations to this study. First, the experiment with the existing dataset that was different from finding information in a real environment. Therefore, more work must be performed to know whether the results hold true in the real world. Second, this study was conducted using a movie dataset. For a general of research results, further study is required using the dataset from various domains. Third, the algorithm used in the experiment is a deep neural network-based algorithm that is commonly used in the study of recommendation systems. We are not sure whether other algorithms, such as Recurrent Neural Network (RNN) or Convolutional Neural Network (CNN) would result in the same findings. Therefore, further research is needed on how the results of this study will appear when various algorithms are used.

Author Contributions: Conceptualization, I.C. and Q.L.; Methodology, I.C. and Q.L.; Data curation, Q.L. and J.K.;Writing, Q.L.; Supervision, J.K.

Funding: This research received no external funding.

Conflicts of Interest: The authors declare no conflict of interest.

\section{References}

1. Linden, G.; Smith, B.; York, J., Amazon. com recommendations: Item-to-item collaborative filtering. IEEE Internet computing 2003, (1), 76-80.

2. Bennett, J.; Lanning, S. In The netflix prize, Proceedings of KDD cup and workshop, 2007; New York, NY, USA.: 2007; p 35.

3. Das, A. S.; Datar, M.; Garg, A.; Rajaram, S. In Google news personalization: scalable online collaborative filtering, Proceedings of the 16th international conference on World Wide Web, 2007; ACM: 2007; pp 271-280.

4. Guo, Y.; Yin, C.; Li, M.; Ren, X.; Liu, P., Mobile e-commerce recommendation system based on multi-source information fusion for sustainable e-business. Sustainability 2018, 10, (1), 147.

5. Kumar, N.; Benbasat, I., Research note: the influence of recommendations and consumer reviews on evaluations of websites. Information Systems Research 2006, 17, (4), 425-439.

6. Xiao, B.; Benbasat, I., E-commerce product recommendation agents: use, characteristics, and impact. MIS quarterly 2007, 31, (1), 137-209.

7. Thirumalai, S.; Sinha, K. K., Customization strategies in electronic retailing: Implications of customer purchase behavior. Decision Sciences 2009, 40, (1), 5-36.

8. Herlocker, J. L.; Konstan, J. A.; Terveen, L. G.; Riedl, J. T., Evaluating collaborative filtering recommender systems. ACM Transactions on Information Systems (TOIS) 2004, 22, (1), 5-53.

9. $\mathrm{Pu}, \mathrm{P}$;; Chen, L.; Hu, R. In A user-centric evaluation framework for recommender systems, Proceedings of the fifth ACM conference on Recommender systems, 2011; ACM: 2011; pp 157-164.

10. Cho, Y. H.; Kim, J. K., Application of Web usage mining and product taxonomy to collaborative recommendations in e-commerce. Expert systems with Applications 2004, 26, (2), 233-246.

11. Cho, Y. H.; Kim, J. K.; Kim, S. H., A personalized recommender system based on web usage mining and decision tree induction. Expert systems with Applications 2002, 23, (3), 329-342. 
12. Herlocker, J. L.; Konstan, J. A.; Riedl, J. In Explaining collaborative filtering recommendations, Proceedings of the 2000 ACM conference on Computer supported cooperative work, 2000; ACM: 2000; pp 241-250.

13. Lee, J.; Bengio, S.; Kim, S.; Lebanon, G.; Singer, Y. In Local collaborative ranking, Proceedings of the $23 \mathrm{rd}$ international conference on World wide web, 2014; ACM: 2014; pp 85-96.

14. Shardanand, U.; Maes, P. In Social information filtering: algorithms for automating" word of mouth", Chi, 1995; Citeseer: 1995; pp 210-217.

15. Zhu, W.; Lu, J.; Li, Y.; Yang, Y., A Pick-Up Points Recommendation System for Ridesourcing Service. Sustainability 2019, 11, (4), 1097.

16. Son, J.; Kim, S. B.; Kim, H.; Cho, S., Review and analysis of recommender systems. Journal of Korean Institute of Industrial Engineers 2015, 41, (2), 185-208.

17. Lathia, N.; Hailes, S.; Capra, L.; Amatriain, X. In Temporal diversity in recommender systems, Proceedings of the 33rd international ACM SIGIR conference on Research and development in information retrieval, 2010; ACM: 2010; pp 210-217.

18. Goldberg, D.; Nichols, D.; Oki, B. M.; Terry, D., Using collaborative filtering to weave an information tapestry. Communications of the ACM 1992, 35, (12), 61-71.

19. Lekakos, G.; Giaglis, G. M., Improving the prediction accuracy of recommendation algorithms: Approaches anchored on human factors. Interacting with computers 2006, 18, (3), 410-431.

20. Resnick, P.; Iacovou, N.; Suchak, M.; Bergstrom, P.; Riedl, J. In GroupLens: an open architecture for collaborative filtering of netnews, Proceedings of the 1994 ACM conference on Computer supported cooperative work, 1994; ACM: 1994; pp 175-186.

21. Su, X.; Khoshgoftaar, T. M., A survey of collaborative filtering techniques. Advances in artificial intelligence 2009, 2009.

22. Cho, K.; Van Merriënboer, B.; Gulcehre, C.; Bahdanau, D.; Bougares, F.; Schwenk, H.; Bengio, Y., Learning phrase representations using RNN encoder-decoder for statistical machine translation. arXiv preprint arXiv:1406.1078 2014.

23. Girshick, R. In Fast r-cnn, Proceedings of the IEEE international conference on computer vision, 2015; 2015; pp 1440-1448.

24. Ren, S.; He, K.; Girshick, R.; Sun, J. In Faster r-cnn: Towards real-time object detection with region proposal networks, Advances in neural information processing systems, 2015; 2015; pp 91-99.

25. Cheng, H.-T.; Koc, L.; Harmsen, J.; Shaked, T.; Chandra, T.; Aradhye, H.; Anderson, G.; Corrado, G.; Chai, W.; Ispir, M. In Wide $\mathcal{E}$ deep learning for recommender systems, Proceedings of the 1st workshop on deep learning for recommender systems, 2016; ACM: 2016; pp 7-10.

26. Covington, P.; Adams, J.; Sargin, E. In Deep neural networks for youtube recommendations, Proceedings of the 10th ACM conference on recommender systems, 2016; ACM: 2016; pp 191-198.

27. Cremonesi, P.; Garzotto, F.; Negro, S.; Papadopoulos, A. V.; Turrin, R. In Looking for "good" recommendations: A comparative evaluation of recommender systems, IFIP Conference on Human-Computer Interaction, 2011; Springer: 2011; pp 152-168.

28. Hidasi, B.; Karatzoglou, A.; Baltrunas, L.; Tikk, D., Session-based recommendations with recurrent neural networks. arXiv preprint arXiv:1511.06939 2015.

29. Van den Oord, A.; Dieleman, S.; Schrauwen, B. In Deep content-based music recommendation, Advances in neural information processing systems, 2013; 2013; pp 2643-2651.

30. Devooght, R.; Bersini, H., Collaborative filtering with recurrent neural networks. arXiv preprint arXiv:1608.07400 2016.

31. He, X.; Liao, L.; Zhang, H.; Nie, L.; Hu, X.; Chua, T.-S. In Neural collaborative filtering, Proceedings of the 26th international conference on world wide web, 2017; International World Wide Web Conferences Steering Committee: 2017; pp 173-182.

32. Oliver, R. L., Effect of expectation and disconfirmation on postexposure product evaluations: An alternative interpretation. Journal of applied psychology 1977, 62, (4), 480.

33. Athiyaman, A., Linking student satisfaction and service quality perceptions: the case of university education. European journal of marketing 1997, 31, (7), 528-540.

34. Bitner, M. J., Evaluating service encounters: the effects of physical surroundings and employee responses. Journal of marketing 1990, 54, (2), 69-82. 
35. Chong, B.; Wong, M., Crafting an effective customer retention strategy: a review of halo effect on customer satisfaction in online auctions. International Journal of Management and Enterprise Development 2005, 2, (1), 1226.

36. Maxham III, J. G., Service recovery's influence on consumer satisfaction, positive word-of-mouth, and purchase intentions. Journal of business research 2001, 54, (1), 11-24.

37. Liang, T.-P.; Lai, H.-J.; Ku, Y.-C., Personalized content recommendation and user satisfaction: Theoretical synthesis and empirical findings. Journal of Management Information Systems 2006, 23, (3), 45-70.

38. Herlocker, J. L.; Konstan, J. A.; Borchers, A.; Riedl, J. In An algorithmic framework for performing collaborative filtering, 22nd Annual International ACM SIGIR Conference on Research and Development in Information Retrieval, SIGIR 1999, 1999; Association for Computing Machinery, Inc: 1999; pp 230-237.

39. Wu, Y.-H.; Chen, A. L. In Index structures of user profiles for efficient web page filtering services, Proceedings 20th IEEE International Conference on Distributed Computing Systems, 2000; IEEE: 2000; pp 644-651.

40. Wartena, C.; Slakhorst, W.; Wibbels, M.; Gantner, Z.; Freudenthaler, C.; Newell, C.; Schmidt-Thieme, L. In Keyword-Based TV Program Recommendation, ITWP@ IJCAI, 2011; 2011.

41. Balabanović, M.; Shoham, Y., Fab: content-based, collaborative recommendation. Communications of the ACM 1997, 40, (3), 66-72.

42. Bengio, Y.; Courville, A.; Vincent, P., Representation learning: A review and new perspectives. IEEE transactions on pattern analysis and machine intelligence 2013, 35, (8), 1798-1828.

43. Schmidhuber, J., Deep learning in neural networks: An overview. Neural networks 2015, 61, 85-117.

44. Szegedy, C.; Toshev, A.; Erhan, D. In Deep neural networks for object detection, Advances in neural information processing systems, 2013; 2013; pp 2553-2561.

45. Nair, V.; Hinton, G. E. In Rectified linear units improve restricted boltzmann machines, Proceedings of the 27 th international conference on machine learning (ICML-10), 2010; 2010; pp 807-814.

46. Hinton, G. E., A practical guide to training restricted Boltzmann machines. In Neural networks: Tricks of the trade, Springer: 2012; pp 599-619.

47. LeCun, Y.; Bottou, L.; Bengio, Y.; Haffner, P., Gradient-based learning applied to document recognition. Proceedings of the IEEE 1998, 86, (11), 2278-2324.

48. Mikolov, T.; Karafiát, M.; Burget, L.; Černocký, J.; Khudanpur, S. In Recurrent neural network based language model, Eleventh annual conference of the international speech communication association, 2010; 2010.

49. Sainath, T. N.; Mohamed, A.-r.; Kingsbury, B.; Ramabhadran, B. In Deep convolutional neural networks for LVCSR, 2013 IEEE international conference on acoustics, speech and signal processing, 2013; IEEE: 2013; pp 8614-8618.

50. McCollough, M. A.; Berry, L. L.; Yadav, M. S., An empirical investigation of customer satisfaction after service failure and recovery. Journal of service research 2000, 3, (2), 121-137.

51. Audrain-Pontevia, A.-F.; Balague, C., The relationships between dissatisfaction, complaints and subsequent behavior in electronic marketplace. ACR North American Advances 2008.

52. Lu, Y.; Lu, Y.; Wang, B., Effects of dissatisfaction on customer repurchase decisions in e-commerce-an emotion-based perspective. Journal of Electronic Commerce Research 2012, 13, (3), 224.

53. Bhattacherjee, A., Understanding information systems continuance: an expectation-confirmation model. MIS quarterly 2001, 351-370.

54. Roca, J. C.; Chiu, C.-M.; Martínez, F. J., Understanding e-learning continuance intention: An extension of the Technology Acceptance Model. International Journal of human-computer studies 2006, 64, (8), 683-696.

55. Mudambi, S. M.; Schuff, D., What makes a helpful review? A study of customer reviews on Amazon. com. MIS quarterly 2010, 34, (1), 185-200.

56. Goldberg, K.; Roeder, T.; Gupta, D.; Perkins, C., Eigentaste: A constant time collaborative filtering algorithm. information retrieval 2001, 4, (2), 133-151.

57. Calvo-Porral, C.; Lévy-Mangin, J.-P., Switching behavior and customer satisfaction in mobile services: Analyzing virtual and traditional operators. Computers in Human Behavior 2015, 49, 532-540.

58. Deng, Z.; Lu, Y.; Wei, K. K.; Zhang, J., Understanding customer satisfaction and loyalty: An empirical study of mobile instant messages in China. International journal of information management 2010, 30, (4), 289-300.

59. Gerpott, T. J.; Rams, W.; Schindler, A., Customer retention, loyalty, and satisfaction in the German mobile cellular telecommunications market. Telecommunications policy 2001, 25, (4), 249-269.

60. Abdel-Hafez, A.; Tang, X.; Tian, N.; Xu, Y. In A reputation-enhanced recommender system, International Conference on Advanced Data Mining and Applications, 2014; Springer: 2014; pp 185-198. 
61. Christoffel, F.; Paudel, B.; Newell, C.; Bernstein, A. In Blockbusters and wallflowers: Accurate, diverse, and scalable recommendations with random walks, Proceedings of the 9th ACM Conference on Recommender Systems, 2015; ACM: 2015; pp 163-170.

62. Zhou, T.; Kuscsik, Z.; Liu, J.-G.; Medo, M.; Wakeling, J. R.; Zhang, Y.-C., Solving the apparent diversityaccuracy dilemma of recommender systems. Proceedings of the National Academy of Sciences 2010, 107, (10), 4511-4515.

63. McGinty, L.; Smyth, B. In On the role of diversity in conversational recommender systems, International Conference on Case-Based Reasoning, 2003; Springer: 2003; pp 276-290.

64. McNee, S. M.; Riedl, J.; Konstan, J. A. In Being accurate is not enough: how accuracy metrics have hurt recommender systems, CHI'06 extended abstracts on Human factors in computing systems, 2006; ACM: 2006; pp 1097-1101.

65. Smyth, B.; McClave, P. In Similarity vs. diversity, International conference on case-based reasoning, 2001; Springer: 2001; pp 347-361.

66. Ziegler, C.-N.; McNee, S. M.; Konstan, J. A.; Lausen, G. In Improving recommendation lists through topic diversification, Proceedings of the 14th international conference on World Wide Web, 2005; ACM: 2005; pp 22-32.

67. Zins, A. H.; Bauernfeind, U.; Del Missier, F.; Venturini, A.; Rumetshofer, H., An experimental usability test for different destination recommender systems. na: 2004.

68. Ekstrand, M. D.; Harper, F. M.; Willemsen, M. C.; Konstan, J. A. In User perception of differences in recommender algorithms, Proceedings of the 8th ACM Conference on Recommender systems, 2014; ACM: 2014; pp 161-168. 